\title{
A New Hydrodynamic Equation for Ion-Implantation Simulation
}

\author{
Shiroo Kamohara, Megumi Kawakami \\ Semiconductor Development Center, \\ Semiconductor \& Integrated Circuit Div., Hitachi, Ltd., \\ 1-280, Higashi-koigakubo Kokubunji, Tokyo 185, Japan
}

\begin{abstract}
In this paper, we propose a new hydrodynamic equation that reduces the simulation CPU time of the ion implantation to the complicated multi-layer structures. To simplify introduction of the hydrodynamic equation we introduced a BTE that includes the Fokker-Planch collision term and transformed this BTE into a hierarchical structure by moment expansion. We successfully' shortened the CPU time by a factor of more than 100 .
\end{abstract}

\section{Introduction}

As device structures become more complicated, implanted ions have become subject to more complicated physical phenomena, in which these ions pass through complicated multilayer structures at an arbitrary angle and energy. To simulate these phenomena in two dimension (2D), it is necessary to change the simulation method from an analytical approach [1] using the Boltzmann transport equation (BTE) to other approaches. One is the Monte Carlo approach[2,3] and the other is solving numerically the five dimensional BTE $[4,5,6]$. However, both of these approaches require a prohibitive amount of the CPU time. Depending on circumstances, the CPU time for ion implantation can dominate the total process simulation.

In this paper, we propose a new hydrodynamic equation for analyzing the complicated physical phenomena that also reduces the CPU time for ion implantation simulation. Using this equation, we successfully shortened the CPU time by a factor of more than 100 by reducing the analysis space from a four-dimensional phase space to a two-dimensional coordinate space for $2 \mathrm{D}$ simulation.

\section{BTE Including the Fokker-Plank Collision Term}

Rosenbluth and his co-workers introduced the BTE for a two-body collision problem, in which two particles interact with the coulomb potential [7]. Based on this method, a twobody collision problem with the Moliere potential interaction is expressed as

$$
\begin{aligned}
& \frac{\partial f}{\partial t}=\left(L_{x}+L_{v}\right) f, \\
& L_{x}=-v_{i} \frac{\partial}{\partial x_{i}},
\end{aligned}
$$




$$
L_{v}=\Gamma_{a} N_{b} \frac{m_{a}}{m_{a b}} \frac{\partial}{\partial v_{i}} \frac{v_{i}}{g^{3}}+\frac{\Gamma_{a} N_{b}}{2} \frac{\partial}{\partial v_{i} \partial v_{j}}\left(\frac{g^{2} \delta_{i, j}-v_{i} v_{j}}{g^{3}}\right)
$$

where $\mathrm{m}_{\mathrm{ab}}, \mathrm{g}$, and $\mathrm{G}_{\mathrm{a}}$ are expressed as

$$
\begin{aligned}
& m_{a b}=\frac{m_{a} m_{b}}{m_{a}+m_{b}}, \\
& g=\sqrt{\sum v_{i}^{2}}, \\
& \Gamma_{a}=\frac{8 \pi Z_{a}^{2} Z_{b}^{2} e^{2}}{m_{a}^{2}} \int \phi(s) d s .
\end{aligned}
$$

Here, $\mathrm{f}$ is the phase space distribution function of the implanted ion, $\mathrm{v}$ is the velocity component, $\mathrm{N}$ is the concentration of the atom, $\mathrm{m}$ is the mass, $\mathrm{Z}$ is the atomic number, $\mathrm{e}$ is the elementary charge, $\phi$ is the collision function originating from the Moliere potential [8], $t$ is the time, and $\mathrm{x}$ is the space component. Here, the subscripts a and $\mathrm{b}$ express the implanted ion and the atom in the target, respectively, and the subscript $i$, which obeys the Einstein rule, expresses the direction. While the $L_{V}$ term of Eq. (3) describes only nuclear collisions, implanted ions also suffer electronic collisions. An electronic collision term can be added to Eq. (3) using the same analogy as the nuclear one. Assuming electronic collisions are not extremely dominant during the ion movement, $\mathrm{g}$ - which corresponds to the energy - changes slower than each of the velocity components. Under this assumption, we can place g outside of the differential operator. Then, we can approximately transform Eq. (3) into Eqs. (7) expressed as

$$
L_{v} \approx \gamma \frac{\partial}{\partial v_{i}} v_{i}+\gamma_{n} k \frac{\partial}{\partial v_{i} \partial v_{j}}\left(g^{2} \delta_{i, j}-v_{i} v_{j}\right)
$$

where

$$
\begin{aligned}
& k=\frac{1}{2} \frac{m_{a b}}{m_{a}}, \\
& \gamma=\gamma_{n}+\gamma_{e}, \\
& \gamma_{n}=\Gamma_{a} N_{b} \frac{m_{a}}{m_{a b}} \frac{1}{g^{3}}=\frac{m_{b}}{m_{a b} m_{a}} \frac{S_{n}(g)}{g}, \\
& \gamma_{e}=\frac{m_{b}}{m_{a b} m_{a}} \frac{S_{e}(g)}{g} .
\end{aligned}
$$

Here $\gamma$ is the collision cofficient, $\mathrm{S}$ is the stopping power, and $\delta$ is the Kronecker delta function. The subscript e and $\mathrm{n}$, respectively denote an electronic collision and a nuclear collision. Using Eqs. (1), (2), and (7), we can analyze the time evolution of the implanted ion in the phase space. This BTE which describes the implanted ion dynamics makes the moment expansion the same as that generally used in fluid dynamics.

\section{Hydrodynamic Equation for Ion Implantation}


To reduce the analysis time of our BTE, we expand the BTE by the moments. The zero order moment is obtained from the $v_{1}$ and $v_{2}$ integration of Eq. (1). The 1 st order moment of $v_{1}$ is obtained from the $v_{1}$ and $v_{2}$ integration of Eq. (1) after multiplying $v_{1}$ by both sides of Eq. (1). The higher order moments are also obtained in the same way. The integration does not operate on $\gamma$ and $\gamma_{n}$, because these parameters change more slowly than the change of the phase space distribution function under our assumption. By these moment expansions, we obtained the simultaneous differential equation expressed as,

$$
\begin{aligned}
& \frac{\partial C_{0,0}}{\partial \mathrm{t}}=-\frac{\partial C_{1,0}}{\partial x_{1}}-\frac{\partial C_{0.1}}{\partial x_{2}}, \\
& \frac{\partial C_{1,0}}{\partial \mathrm{t}}=-\gamma C_{1,0}-\frac{\partial C_{2,0}}{\partial x_{1}}-\frac{\partial C_{1.1}}{\partial x_{2}}, \\
& \frac{\partial C_{0,1}}{\partial \mathrm{t}}=-\gamma C_{0,1}-\frac{\partial C_{1.1}}{\partial x_{1}}-\frac{\partial C_{0,2}}{\partial x_{2}}, \\
& \frac{\partial C_{1,1}}{\partial \mathrm{t}}=-2 \gamma C_{1,1}-2 \gamma_{n} k C_{1,1}-\frac{\partial C_{0,1} C_{2,0} / C_{0,0}}{\partial x_{1}}-\frac{\partial C_{1,0} C_{0,2} / C_{0,0}}{\partial x_{2}}, \\
& \frac{\partial C_{2,0}}{\partial \mathrm{t}}=-2 \gamma C_{2.0}+2 \gamma_{n} k C_{0,2}-\frac{\partial C_{1,0} C_{2,0} / C_{0,0}}{\partial x_{1}}-\frac{\partial C_{0,1} C_{2,0} / C_{0,0}}{\partial x_{2}}, \\
& \frac{\partial C_{0.2}}{\partial \mathrm{t}}=-2 \gamma C_{0,2}+2 \gamma_{n} k C_{2,0}-\frac{\partial C_{1,0} C_{0,2} / C_{0,0}}{\partial x_{1}}-\frac{\partial C_{0.1} C_{0,2} / C_{0,0}}{\partial x_{2}},
\end{aligned}
$$

where $\mathrm{C}_{1,0}=\left\langle\mathrm{v}_{1}\right\rangle, \mathrm{C}_{0,1}=\left\langle\mathrm{v}_{2}\right\rangle, \mathrm{C}_{2,0}=\left\langle\mathrm{v}_{1} 2\right\rangle, \mathrm{C}_{0,2}=\left\langle\mathrm{v}_{2}{ }^{2}>\right.$ and, $\mathrm{C}_{1,1}=\left\langle\mathrm{v}_{1} \mathrm{v}_{2}\right\rangle$. Here, subscripts 1 and 2 denote two directions in the two dimensional coordinate space. We approximate the $3 \mathrm{rd}$ order moment by using $C_{2,1}=C_{0,1} C_{2,0} / C_{0,0}, C_{1,2}=C_{1,0} C_{0,2} / C_{0,0}$, $C_{3,0}=C_{1,0} C_{2,0} / C_{0.0}$, and $C_{0,3}=C_{0,1} C_{0,2} / C_{0,0}$, where $\left.C_{2,1}=\left\langle\mathrm{v}_{1} \mathrm{v}_{1} \mathrm{v}_{2}\right\rangle, \mathrm{C}_{1,2}=<\mathrm{v}_{1} \mathrm{v}_{2} \mathrm{v}_{2}\right\rangle$, $\mathrm{C}_{3,0}=\left\langle\mathrm{v}_{1} \mathrm{v}_{1} \mathrm{v}_{1}\right\rangle$ and $\mathrm{C}_{0,3}=\left\langle\mathrm{v}_{2} \mathrm{v}_{2} \mathrm{v}_{2}\right\rangle$. This approximation means the fluctuation of the 3rd order moment is negligible. We verified the accuracy of this assumption by checking the distribution function in velocity space using our in-house simulator [4]. Eq. (12) corresponds to the transport of the particle density. Eq. (13) and Eq. (14) correspond to that of the momentum component density. Eq. (15), Eq. (16) and Eq. (17) correspond to that of the energy density. This new hydrodynamic equation is numerically solved using the CGS method.

\section{Simulation results}

To verify the accuracy of our hydrodynamic equation, we compared the simulation and experimental results for when a $2.0 \times 10^{16} \mathrm{~cm}^{-2}$ dose of arsenic was implanted into a silicon substrate at $30 \mathrm{keV}$ and $50 \mathrm{keV}$, respectively. Good agreement is obtained as shown in Fig. 1. The CPU time for these simulations with 1000 mesh points is $6.6 \mathrm{sec}$ when using the HITACHI M880 mainframe computer. Figure 2 shows the calculation results in which a $3.0 \times 10^{15} \mathrm{~cm}^{-2}$ dose of boron is implanted into a trench-shaped silicon substrate at $100 \mathrm{keV}$. The CPU time of this simulation for 2500 mesh points is $134 \mathrm{sec}$. Table 1 lists the CPU time of the previous approaches and our approach. This confirms that our approach is 100 times faster than the conventional approach by reducing the analysis space from a fourdimensional phase space to a two-dimensional coordinate space. 


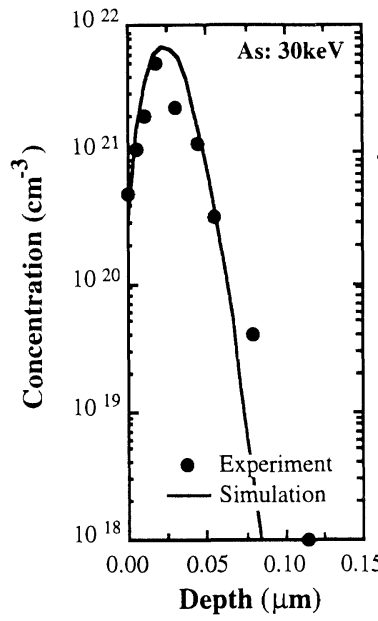

(a)

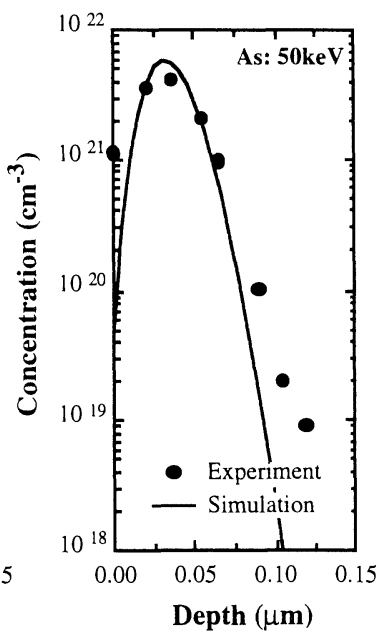

(b)

Fig. 1. Comparisons of the simulation and the experimental results for a $2.0 \times 10^{16} \mathrm{~cm}^{-2}$ dose of As.

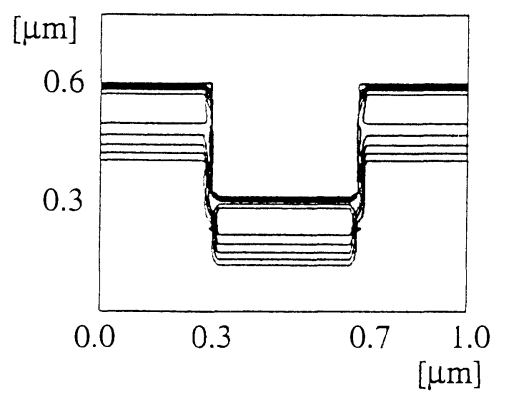

Fig. 2. Contour plot of the $3.0 \times 10^{15} \mathrm{~cm}^{-2}$ dose of B implanted into a trench-shaped silicon substrate.

Table 1. CPU-time comparisons
for ion implantation into a planar
silicon substrate.
Method
This Work
Monte Carlo ${ }^{* 2}$
Numerical ${ }^{* 3}$
*1: HTACHI (min.)
*2: Our in-house simulator.
(The method is the same as that of ref. [3] )
*3: Our in-house simulator [5].

\section{References}

[1]J. Linhard, M. Scharff and H. E. Schiott, Vol. 33, No. 14, pp. 3-42, 1963.

[2]T. Ishitani, R. Shimizu and K. Murata, Jpn. J. Appl. Phys., Vol. 11, pp. 125-133, 1972.

[3]T. Ishitani, R. Shimizu and K. Murata, Phys. stat. sol., Vol. 50, pp. 681-690, 1972.

[4]I. Saitoh, and N. Natsuaki, VLSI Process/Device Modeling Workshop, pp. 102-103, 1990.

[5]L. A. Christel and J. F. Gibbons, S. Mylroie, J. Appl. Phys., Vol. 51, pp. 6176-6182, 1980.

[6]T. Takeda and A. Yoshii, IEEE Electron Device Letters, Vol. EDL-4, pp. 430-432, 1983. [7]M. N. Rosenbluth, W. M. MacDonald, and D. L. Judo, Phys. Rev. Vol. 107, pp. 1-6, 1957.

[8]G. P. Mueller, Radiat. Eff. Lett., Vol. 50, pp.87-92, 1980. 